\title{
Nonwoven Membrane Supports from Renewable Resources: Bamboo Fiber Reinforced Poly(Lactic Acid) Composites
}

Hai Anh Le Phuonga, ${ }^{\mathrm{a}, \mathrm{b}, \#}$, Nor Amira Izzati Ayob ${ }^{\mathrm{c}, \#}$, Christopher F. Blanford ${ }^{\mathrm{b}, \mathrm{d}}$, Nurul Fazita Mohammad Rawic, Gyorgy Szekely ${ }^{\mathrm{a}, \mathrm{e}, *}$

a School of Chemical Engineering \& Analytical Science, The University of Manchester, The Mill, Sackville street, Manchester, M13 9PL, United Kingdom

${ }^{b}$ School of Materials, University of Manchester, Oxford Road, Manchester, M13 9PL, United Kingdom

' Division of Bioresource, Paper and Coatings Technology, School of Industrial Technology, Universiti Sains

Malaysia, Penang, 11800, Malaysia

${ }^{\mathrm{d}}$ Manchester Institute of Biotechnology, University of Manchester, 131 Princess Street, Manchester, M1

7DN, United Kingdom

e King Abdullah University of Science and Technology (KAUST), Advanced Membranes and Porous Materials

Center (AMPMC), Thuwal, 23955-6900, Saudi Arabia

${ }^{*}$ Corresponding author

"These authors contributed equally.

Tel.: +441613064366; +966128082769

E-mail: gyorgy.szekely@manchester.ac.uk; gyorgy.szekely@kaust.edu.sa

\section{Keywords}

composite membrane, biodegradable material, sustainability, green solvent, filtration

\section{Table of Contents Graphic}

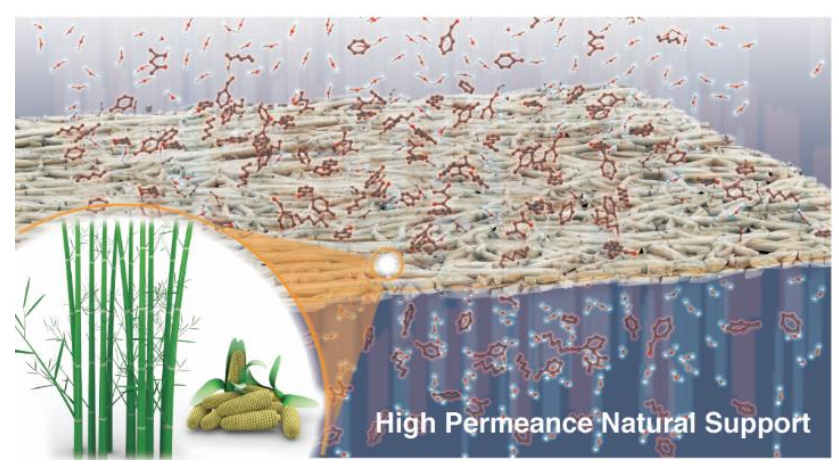




\begin{abstract}
Membrane separation has been widely recognized as an energy-efficient technology with a rapidly growing market. However, such growth raises concerns about sustainability due to current fabrication methods that employ toxic solvents and non-degradable petroleum-based polymers. The focus of tackling these challenges has been on the active layer of membranes via renewable materials, while the equally important membrane supports are yet to turn green. Herein we report the fabrication of sustainable, biodegradable, non-woven composite membrane supports made from three renewable materials: bamboo fiber, poly(lactic acid) (PLA) and dimethyl carbonate. The bio-based membrane supports exhibited a porous structure (porosity of $0.719 \pm 0.132$ ) with tensile strength $(32.7-73.3 \mathrm{MPa}$ ) comparable to conventional materials, such as polypropylene. The microstructure and porosity of the supports were revealed by laser scanning confocal microscopy. The increase in bamboo content resulted in increased mechanical stability, decreased swelling and enhanced permeance, up to $1068 \pm 32 \mathrm{~L} \mathrm{~m}^{-2} \mathrm{~h}^{-1} \mathrm{bar}^{-1}$ in water. The long-term chemical stability of membrane supports was verified in 19 of the 25 organic solvents screened. In particular, they were found to be stable in some conventional and emerging green polar aprotic solvents including Cyrene, 2-methyltetrahydrofuran, $\gamma$-valerolactone and propylene carbonate. Stable cross-flow filtration performance over 2 weeks was successfully demonstrated. The results demonstrated that the bamboo/PLA membrane supports could provide a sustainable alternative for conventional membrane backing materials by eliminating the need for petroleum-based non-degradable polymers and toxic solvents.
\end{abstract}

\title{
Introduction
}

Membrane separation has been recognized as a powerful tool for process intensification due to its mild operating conditions, energy efficiency and modular nature. ${ }^{1}$ Membrane technology, therefore, has grown rapidly in the past 40 years and has been used in different industrial sectors with applications in desalination, food processing, petrochemical and pharmaceutical industries. Although, membrane separation has been widely investigated as a sustainable alternative or complementing unit operation to distillation and chromatography, sustainable membrane fabrication is yet to be solved before the overall membrane filtration can be considered green. ${ }^{2,3}$ Membrane processes will only surpass distillation in terms of sustainability when more than $100 \mathrm{~L}$ of solvent per $\mathrm{m}^{2}$ membrane area has been processed. ${ }^{2}$ Membrane fabrication usually requires toxic polar aprotic solvents, such as $\mathrm{N}, \mathrm{N}$-dimethyl acetamide, $\mathrm{N}, \mathrm{N}$-dimethyl formamide or $\mathrm{N}$-methyl pyrrolidone, and generates large amount of wastewater estimated to be approximately 50 billion liters annually. ${ }^{4}$ In addition, membranes, both active layers and non-woven membrane supports, are currently prepared from non-renewable, petroleum-based polymer materials, such as polyimide, aromatic polyamide, polyethylene terephthalate (PET), polyethylene (PE) and polypropylene (PP), which are incinerated at the end of their life.

There are several strategies to improve the sustainability of membrane fabrication. Toxic solvents can be replaced by greener alternatives, such as PolarClean, ${ }^{4}$ ionic liquids, ${ }^{5} \psi$-valerolactone ${ }^{6}$ or water, ${ }^{7}$ to prepare the active layer of membranes. Furthermore, the wastewater generated from membrane fabrication can be treated and recycled for further membrane preparation. ${ }^{3}$ In order to mitigate the environmental burden posed by membrane fabrication, another feasible strategy is to prepare biodegradable membranes from renewable materials that do not persist in the environment. Biodegradable membranes from renewable materials including cellulose acetate, polyhydroxyalkanoates, chitosan and collagen mainly for applications such as desalination, pervaporation and gas separation, have been recently reported. ${ }^{8}$ The focal point of the 
renewable materials research has been on the active layer of the membrane, and the equally important membrane supports are yet to turn green.

Poly(lactic acid) (PLA), a polyester derived from renewable biomass, usually from fermented maize starch, is one of the most widely investigated and commercially used biodegradable polymer. PLA degrades through hydrolysis when exposed to moisture after several months, making it attractive as a green alternative for petroleum-based polymers. ${ }^{9}$ Previously, PLA was mainly used for health and biomedical science due to its limited availability. However, with the introduction of new technologies and effective production of PLA from renewable sources,$^{10}$ the application of PLA to other fields, including membrane fabrication, has been expanding. ${ }^{11}$ In addition to the renewability and degradability, PLA has the advantage of better thermal processability compared to other biopolymers as well as tensile strength and elastic modulus comparable to PET. Moreover, the production of PLA requires $25-55 \%$ less energy compared to petrochemical-based polymers, ${ }^{9}$ therefore, could provide an alternative for the fabrication of biodegradable, green membrane supports. However, the low thermal stability (thermal degradation occurring above $200{ }^{\circ} \mathrm{C}$ ), low toughness and brittle nature of PLA can hinder its large-scale application. ${ }^{9}$ In order to improve the toughness and mechanical stability, fillers such as bamboo fiber, can be introduced within the polymer matrix (Figure 1).

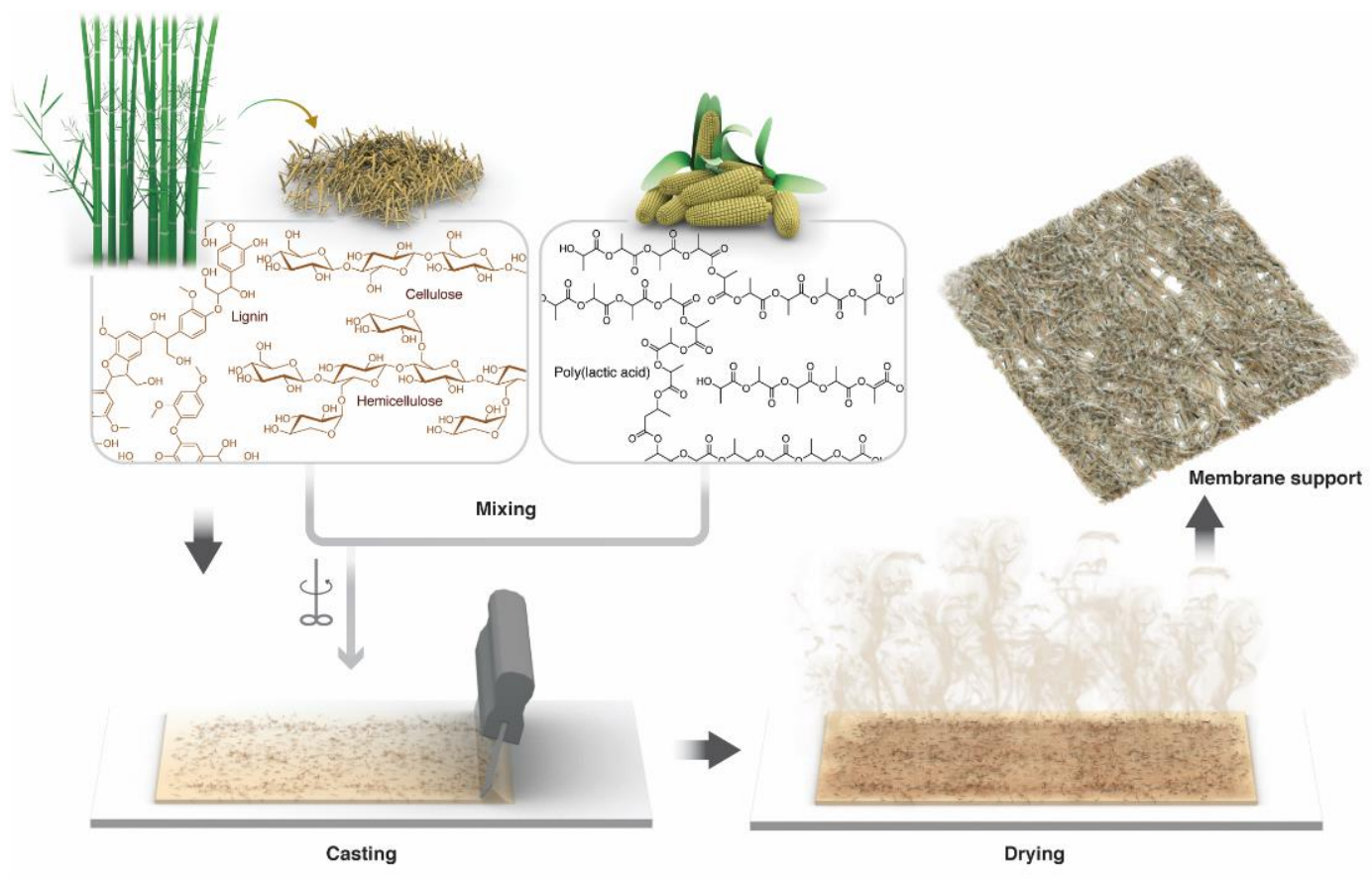

Figure 1. Schematic overview of the membrane support fabrication: dissolution of PLA followed by the addition of bamboo fiber (step 1), casting of the membrane support (step 2), and evaporation of the solvent (step 3).

Natural fibers have been widely investigated as a green filler for the reinforcement of thermoplastics. Compared to their synthetic counterparts, natural fibers are renewable, inexpensive (US\$0.2-1 kg-1) and their production requires $85-95 \%$ lower energy. ${ }^{12}$ Natural fibers have also shown high specific stiffness with comparable mechanical properties to glass or carbon fiber making them attractive alternative for novel materials. ${ }^{13}$ Among the natural fibers, bamboo has been gaining wide interest due to its low cost and abundance in nature with an annual global production of $3 \times 10^{10} \mathrm{~kg} .{ }^{14}$ The use of bamboo fibers as reinforcement in composite materials has increased tremendously, and has undergone high-tech revolution in recent years as a response to the increasing demand for developing biodegradable, sustainable, and recyclable materials. Owing to its low density and acceptable specific mechanical properties provided by the 
longitudinally aligned fibers, bamboo fibers provide an attractive solution for the reinforcement of PLA to provide a completely bio-derived and biodegradable final material. ${ }^{15}$ However, bamboo fibers are hydrophilic due to the large number of hydroxyl groups, which result in poor compatibility with hydrophobic PLA. This phenomenon is perceived as a disadvantage for most applications. On the contrary, herein we propose to exploit the poor interfacial adhesion between the polymer matrix and bamboo fibers, which leads to a porous structure making the bamboo fiber/PLA composite suitable for membrane supports. There has been extensive research on bamboo fiber/PLA composite, albeit most of them solely focused on the improvement of their compatibility and on the characterization of physical and mechanical properties. The aim of these studies was to replace petroleum-based composites in packaging, construction, aircraft interior and flooring materials. ${ }^{16,17,18}$ However, there are no reports on the application of bamboo fiber/PLA composites for membranes.

Herein we report the fabrication of bamboo fiber/PLA composite as a sustainable, biodegradable, nonwoven backing material for polymer membranes (Figure 1). Composite membrane supports were fabricated with bamboo:PLA mass ratios from 0.1 to 2 (Table 1). Their applicability as a backing material was investigated by filtration, mechanical, morphological, chemical characterization methods. To demonstrate the viability and compatibility of the bamboo fiber/PLA composites as membrane supports, polybenzimidazole (PBI) nanofiltration membrane ${ }^{19,20}$ was cast on the membrane supports.

Table 1. Membrane support designations and compositions.

\begin{tabular}{ccccc} 
Membrane Support & $\begin{array}{c}\text { Bamboo }^{a} \\
(\text { wt\% })\end{array}$ & $\begin{array}{c}\text { PLA }^{a} \\
(\text { wt } \%)\end{array}$ & $\begin{array}{c}\text { DMC }^{a} \\
(\text { wt\% })\end{array}$ & $\begin{array}{c}\text { Casting thickness } \\
(\mu \mathrm{m})\end{array}$ \\
S1 & 1 & 10 & 89 & $150^{a} / 100^{b}$ \\
S2 & 5 & 10 & 85 & $150^{a} / 100^{b}$ \\
S3 & 10 & 5 & 85 & $150^{a} / 100^{b}$ \\
S4 & 10 & 10 & 80 & $150^{a} / 100^{b}$ \\
S5 & 10 & 15 & 75 & $150^{a} / 100^{b}$ \\
\hline
\end{tabular}

${ }^{a}$ Bamboo fiber/PLA membrane support fabrication; ${ }^{b} \mathrm{PBI}$ membrane fabrication on the support

\section{Experimental}

\section{Materials}

Poly(lactic acid) (PLA) pellets (IngeoTM biopolymer 2003D) manufactured by NatureWorks LLC, USA were purchased from Innovative Pultrusion Sdn Bhd, Malaysia. The density, specific gravity, glass temperature and melting point of the PLA are $1.25 \mathrm{~g} \mathrm{~cm}^{-3}, 1.24,60^{\circ} \mathrm{C}$ and $146{ }^{\circ} \mathrm{C}$, respectively. The bamboo was purchased from Bamboo Village, Kuala Lumpur, Malaysia in a form of block. Then, the bamboo was cut, ground and sieved to produce dry particles with the size of 50-150 $\mu \mathrm{m}$. Acetone (analytical reagent grade), acetonitrile (MeCN, $\geq 99.9 \%$ ), 1-butanol ( $\geq 99.8 \%)$, chloroform (analytical reagent grade), dichloromethane (DCM, analytical reagent grade), dimethyl sulfoxide (DMSO, analytical reagent grade), abs. ethanol, ethyl acetate (EtOAc, analytical reagent grade), L-ethyl lactate ( $\geq 98 \%)$, hexane (HPLC grade), heptane (HPLC grade), methanol ( $\mathrm{MeOH}, \geq 99.8 \%$ ), toluene (analytical reagent grade), tetrahydrofuran (THF, analytical reagent grade) and toluene (analytical reagent grade) were purchased from Fisher Scientific. Cyrene ${ }^{\mathrm{TM}}$ (dihydrolevoglucosenone, 99\%), dimethyl carbonate (DMC, 99\%), 2-methyltetrahydrofuran (2-MeTHF, $\geq 99 \%$ ), N,N-dimethylformamide (DMF, $\geq 99.8 \%$ ), N,N-dimethylacetamide (DMAc, $\geq 99.5 \%$ ), 1-methyl-2pyrrolidone (NMP, 99\%), propylene carbonate (PC, 99\%) and $\gamma$-valerolactone (99\%) were purchased from Sigma-Aldrich. Cyclopentyl methyl ether (CPME, $\geq 99.9 \%$ ) and isopropanol (IPA, $\geq 99.5 \%$ ) were purchased 
from Acros Organics. Methyl $t$-butyl ether (MTBE, 99\%) was purchased from Alfa Aesar. Polybenzimidazole dope solution (PBI, 26 wt\% in DMAc) was purchased from PBI Performance Products, Inc. (Charlotte, USA). All materials were used without further purification.

\section{Membrane support and membrane fabrication}

Prior to use, the bamboo fibers and the PLA pellets were dried under vacuum at $25{ }^{\circ} \mathrm{C}$ for 24 hours. Table 1 summarizes the designation and composition of the membranes and their supports. Mixtures of 10 $30 \mathrm{wt} \%$ bamboo fiber and 5-15 wt\% PLA were vigorously stirred at $120 \mathrm{rpm}$ in DMC at $60^{\circ} \mathrm{C}$ for 6 hours. The mixture was cast on a glass plate using a film applicator with a casting knife set to a thickness of $150 \mu \mathrm{m}$ and casting speed of $6 \mathrm{~cm} \mathrm{~s}^{-1}$. The film was immediately placed into a vacuum oven at $30^{\circ} \mathrm{C}$ for 24 hours. 22 wt $\%$ $\mathrm{PBI}$ films were cast on the membrane support using a film applicator with a casting knife set to a thickness of $100 \mu \mathrm{m}$ and casting speed of $6 \mathrm{~cm} \mathrm{~s}^{-1}$, followed by phase inversion in deionized water $(15 \mathrm{M} \Omega \mathrm{cm})$ at $22^{\circ} \mathrm{C}$.

\section{General characterization methods}

The surface and cross-sectional structure of the supports were investigated with scanning electron microscopy (SEM). SEM images were acquired using a FEI Quanta 200 ESEM with a tungsten hairpin filament emission gun at an accelerating voltage of $5 \mathrm{kV}$. The cross sections were obtained by breaking a piece of membrane under liquid nitrogen. Membranes were dried in a vacuum desiccator prior to the sample preparation. Samples were prepared by sticking the dried membranes on conductive carbon tape. The polymeric membranes were coated with $2 \mathrm{~nm}$ Pt using CRESSINGTON 108 auto sputter coater to make the samples conductive. The membrane thickness was measured with the ImageJ software using two different cross section images. Three measurements were performed on each image and the membrane thickness was calculated as an average of the six values.

The topology of supports was investigated with white light interferometry using Bruker ContourGT-X 3D Optical Profiler. Samples were prepared by sticking the dried membranes on a glass slide using double-sided tape. The membrane supports were coated with $2 \mathrm{~nm}$ Pt using CRESSINGTON 108 auto sputter coater to make the samples reflective. Three membranes of each type were scanned and analyzed with the ContourGT Analysis software for roughness calculations.

The topology of PBI membrane was investigated with atomic force microscopy (AFM) measurements. AFM images were taken in tapping mode in air using Bruker Multimode 8 Atomic Force Microscope equipment with Bruker TESPA-V2 probe (nominal spring constant of $37 \mathrm{~N} \mathrm{~m}^{-1}$, nominal tip apex radius of 7 $\mathrm{nm})$. Samples were prepared by sticking the dried membranes on a metal specimen disc using double sided tape. Three membranes of each type with an area of $5 \mu \mathrm{m} \times 5 \mu \mathrm{m}$ were scanned and analyzed with the NanoScope Analysis software for roughness calculations.

The tensile test was performed according to ASTM D882-12. The initial grip separation was set at $60 \mathrm{~mm}$ and a crosshead speed of $50 \mathrm{~mm} \mathrm{~min}{ }^{-1}$. Three samples were tested and the tensile properties such as tensile strength and elongation at break were recorded.

The linear swelling of the membrane supports was determined in various solvents by measuring the length of an approximately $1 \mathrm{~cm} \times 1 \mathrm{~cm}$ membrane piece before and after soaked in solvents for 1 week. The length of the membrane supports was measured with calipers having $5 \mu \mathrm{m}$ accuracy. Five pieces were measured, each 10 times, and the average values and standard deviations are reported. The linear swelling 
ratio $(S R)$ is defined as the relative change in the final length of the membrane support in organic solvents in relation to their original dry length $(S R$, Equation 1$)$.

$S R[\%]=\frac{\text { length in solvents-dry length }}{\text { dry length }} 100 \%$

Eq. 1

Fourier-transform infrared (FTIR) spectra of dry membrane samples were recorded using a Bruker Alpha-P ATR-FTIR spectrometer. The spectra were obtained at a resolution of $4 \mathrm{~cm}^{-1}$ as an average of 24 scans.

Thermogravimetric analysis (TGA) was acquired using a TA Instruments Q500 in $\mathrm{N}_{2}$ atmosphere with the ramp rate of $20^{\circ} \mathrm{C} \mathrm{min}^{-1}$.

Water contact angles (WCA) of membranes were determined by using Krüss DSA100. $1 \mu$ l deionized water was injected on membrane surface, and contact angle was obtained by using the sessile drop fitting function in Drop Shape Analysis.

The chemical stability of membranes were assessed by soaking the membrane supports $(0.5 \mathrm{~cm} \times 0.5 \mathrm{~cm})$ in polar aprotic solvents (PC, DMC, Cyrene, $p$-valerolactone, DMSO, MeTHF, THF, DMF, DMAc, MeCN, NMP); alcohols (methanol, ethanol, IPA, 1-butanol); esters (ethyl lactate, EtOAc); ethers (CPME, MTBE); ketone (acetone); alkanes (hexane, heptane); aromatics (toluene); halogenated solvents (DCM, chloroform) and in water for 180 days at room temperature in sealed vials. For the stability test, $1 \mathrm{~mL}$ solvent was used.

\section{Laser scanning confocal microscopy (LSCM)}

Membrane porosity and particle size distribution was analyzed by laser scanning confocal microscopy (LSM 710, Zeiss). Membrane segments $(1 \mathrm{~cm} \times 2 \mathrm{~cm}$ ) were stained by soaking for $2 \mathrm{~d}$ in a solution of fluorescein (Sigma-Aldrich, $1 \mathrm{~g} \mathrm{~L}^{-1}$ in acetone), then drying for $1 \mathrm{~d}$ in a fume cupboard. The sample was excited with a $561 \mathrm{~nm}$ laser line, away from the absorption maximum of the fluorophore to allow greater light penetration into the sample, through a 0.45 NA 10x Plan-Apochromat objective and a 1 Airy Unit pinhole. A volume of $2.55 \times 2.55 \times 0.18 \mathrm{~mm}^{3}$ was scanned with a voxel size of $1.66 \times 1.66 \times 0.5 \mu^{3}$ and a dwell time of $50 \mu \mathrm{s} /$ voxel. Light with wavelengths longer than $566 \mathrm{~nm}$ was collected. The lateral point resolution was $0.6 \mu \mathrm{m}$ and the optical section thickness was $7.5 \mu \mathrm{m}$. The resultant z-stack was analyzed with the Fiji implementation of ImageJ 2.0.0. The stack threshold was set using the Huang, ${ }^{21}$ IsoData ${ }^{22}$ and $\mathrm{Li}^{23}$ algorithms. 3D representations of the membrane were created with the 3D Viewer plugin. ${ }^{24}$ The component particles in the membranes were tabulated using the BoneJ "Particle Analyser" plugin ${ }^{25}$ (volume resampling $=2$, minimum volume $=0 \mu \mathrm{m}^{3}$ ) on representative z-stacks cropped to remove open space above and below the membrane surface. Particles with zero surface area were manually removed from the list. Occupied volume was calculated as the ratio of the sum of the particle volumes over the volume sampled in the zstack. Sphericity, $\Psi$, was calculated for each particle from its enclosed volume, $V_{\text {encl, }}$ and surface area, $A$, according to Equation 2.

$\psi=\frac{\pi^{1 / 3}\left(6 \mathrm{~V}_{\text {encl }}\right)^{2 / 3}}{\mathrm{~A}}$

Eq. 2

Particles were classified as acicular (needle-shaped) for $\Psi \leq 0.588$, equivalent to a prolate spheroid with its major axis ten times longer than its minor axis. Uncertainty values on volume fraction were calculated as the sample standard deviation of three analyses (one per threshold algorithm) of three representative areas.

Pore size distribution was calculated using the Thickness module of the BoneJ plugin ${ }^{26,27}$ on a LSCM zstack resized to create isotropic voxels $1.66 \mu \mathrm{m}$ on each side, which was the minimum pore radius that could 
be measured. The image was thresholded with the Huang algorithm. The first derivative of cumulative pore volume fraction with respect to pore radius was calculated numerically in Origin with no smoothing.

\section{Filtration performance tests}

Filtration performance tests were carried out in a cross-flow filtration apparatus at 1 bar. ${ }^{28}$ In cross-flow filtration, the liquid travelled tangentially over the membrane surface at a $1.6 \mathrm{~L} \mathrm{~min}{ }^{-1}$ linear velocity to mitigate accumulation of the filtration cake layer on the membrane surface. The reported data are mean values obtained from two membrane pieces with an area $(A)$ of $52.8 \mathrm{~cm}^{2}$. The permeance $(P$, Equation 3$)$ was determined as the volume of permeate divided by the transmembrane pressure $(\Delta P)$, membrane area $(A)$ and filtration time $(t)$.

$P\left[\mathrm{~L} \mathrm{~m}^{-2} \mathrm{~h}^{-1} \mathrm{bar}^{-1}\right]=\frac{V}{\Delta P A t}$

Eq. 3

\section{Results \& Discussion}

Figure 2a presents the FTIR of a typical membrane support, PLA, bamboo fiber, and the PBI membrane. The FTIR of PLA and bamboo fibers are in agreement with the IR spectra reported in the literature. ${ }^{29,30}$ The FTIR spectrum of membrane support does not differ from the FTIR spectrum of PLA indicating the weak vibrations within the bamboo fibers. The only change that can be observed between the two FTIR is the ratio of transmittance between the peaks at $1129 \mathrm{~cm}^{-1}$ and $1082 \mathrm{~cm}^{-1}$ and between the peaks at $1044 \mathrm{~cm}^{-1}$ and $1082 \mathrm{~cm}^{-1}$, which increased by $4.7 \%$ and decreased by $5.3 \%$, respectively. The phenomenon can be attributed to the decreased intensity at $1044 \mathrm{~cm}^{-1}$ and $1082 \mathrm{~cm}^{-1}$ due to the characteristic vibration of bamboo between $1000 \mathrm{~cm}^{-1}$ and $1100 \mathrm{~cm}^{-1}$ in addition to the vibration of PLA. The FTIR spectrum of the PBI membrane is in accordance with previous reports in the literature. ${ }^{20}$ The detailed analysis of FTIR spectra can be found in the Supporting Information.
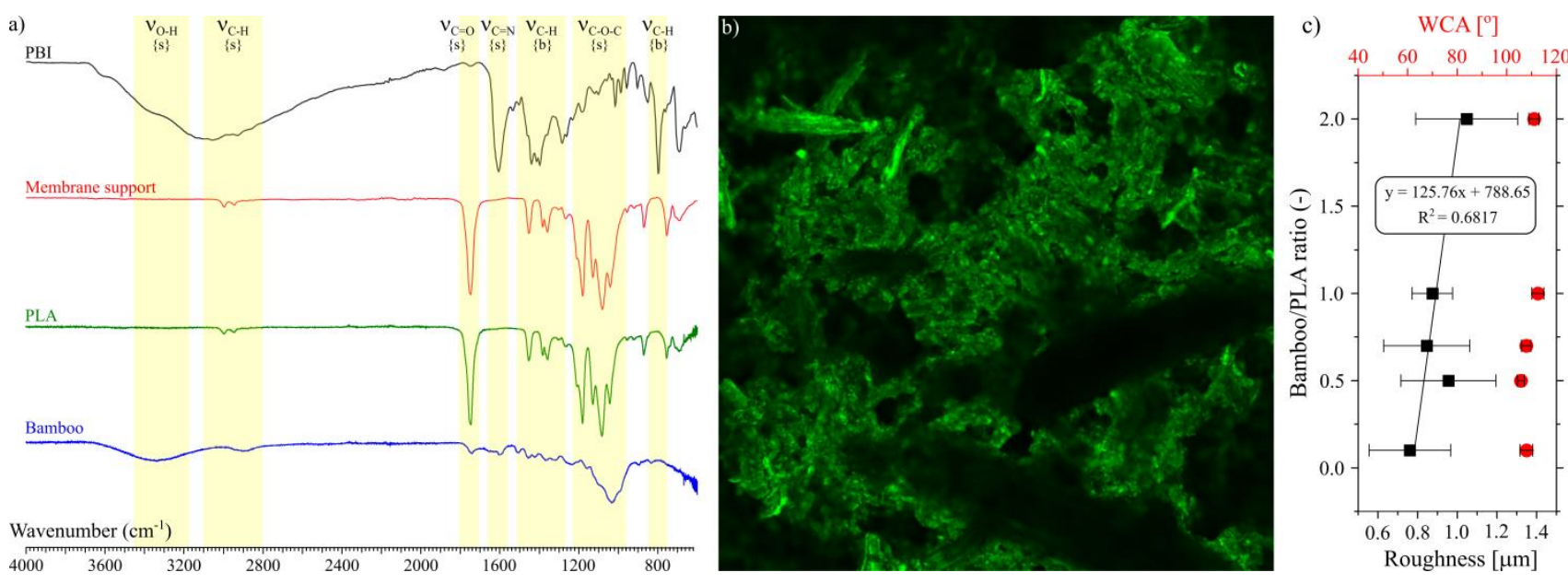

Figure 2. Physicochemical characterization of the different components: a) Typical FTIR spectra of PBI membrane, S3 membrane support, PLA and bamboo fiber. The $\{s\}$ and $\{b\}$ designations correspond to the peaks caused by stretching and bending modes, respectively; b) an LSCM optical section microstructure of fluorescein stained S3 membrane support (image size: $850 \mu \mathrm{m} \times 850 \mu \mathrm{m}$ ); c) root mean square (RMS) roughness and water contact angle of membrane supports as a function of bamboo/PLA mass ratio. 
Membrane porosity, particle size distribution and pore-size distribution were determined by LSCM (Figure 2b). 3D reconstruction of S3 membrane support shows a porous structure with spherical and needle shaped particles. Refer to the Supporting Information for the 3D reconstruction image and video. The analyses of three representative membrane area showed that the bamboo fiber and PLA composite have a porosity of $0.719 \pm 0.132$. Although results showed that $81.3 \pm 6.4 \%$ of particles were spherical (the others were needle-shaped), the volume occupied by needle shaped particles (i.e., bamboo fiber) account for 95.2 $\pm 6.0 \%$. These results are in line with the qualitative appearance (Figure S2 and the supporting video). The pore-size distribution determined from the LSCM data was flat up to about $200 \mu \mathrm{m}$ (Figure S3) as expected for a stochastic fiber network. LSCM analyses showed a minimum pore diameter of $3.3 \mu \mathrm{m}$ and a median of $100 \mu \mathrm{m}$.

The topology and roughness of membrane supports were investigated by white light interferometry (Figure $2 \mathrm{c}$ and Figure S4) instead of AFM. AFM was not suitable because of features taller than $1 \mu \mathrm{m}$. The images of the membrane supports do not show any significant difference in topology (Figure S4a-e). The calculated root mean square (RMS) roughness is shown in Figure $2 \mathrm{c}$. In line with the expectations, the membrane supports exhibit rough surface with RMS roughness values above $700 \mathrm{~nm}$. Although the error of the measurement is relatively large due to the heterogenous and non-woven structure, a subtle increase in RMS roughness as a function of bamboo fiber/PLA ratio can be observed, probably due to the rougher surface of bamboo fibers.

Figure 3 shows the surface and cross section of membrane supports. All membrane top sections provide similar, heterogeneous surface with visible pores. The mass ratio of bamboo fiber/PLA in the membrane supports allows the thickness of the membrane support to be controlled. With increasing bamboo fiber/PLA ratio, the thickness of membrane supports also increases. Within the membrane support, the PLA serves as glue between the bamboo fibers, therefore, increased PLA amount is speculated to result in greater adhesion between the fibers, thus lower membrane thickness. Pure evaporated PLA films yield dense films, ${ }^{31}$ and consequently the bamboo fiber is responsible for obtaining a porous structure. The topology and morphology of PBI membrane were investigated by SEM and AFM (Figure S5). Both SEM and AFM height images show a thin, dense structure (RMS roughness $=0.506 \pm 0.138 \mathrm{~nm}$ ) for the PBI membrane on top of the membrane support. The dense membrane structure is probably the result of delayed demixing due to a decrease in the diffusional exchange rate between solvent and nonsolvent. ${ }^{32}$

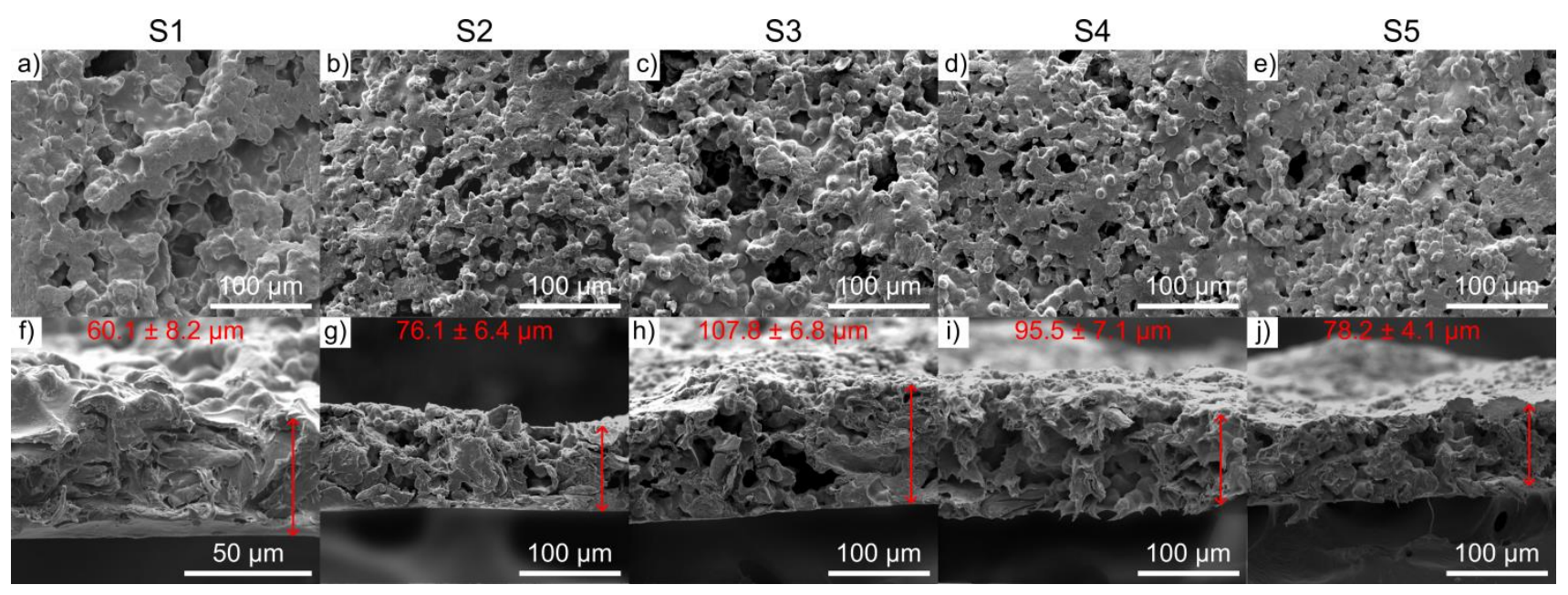

Figure 3. SEM surface (a-e) and cross ( $f-j)$ section images of $S 1-5$ membrane supports. The standard deviation was obtained by measuring the thickness at nine different points. 
The surface wettability was assessed by water contact angle measurements. The contact angle of PLA is reported ${ }^{33}$ to be $69^{\circ}$ and the contact angle of bamboo from Gigantochloa scortechinii is found ${ }^{34}$ to be approximately $15^{\circ}$, therefore the surface of membrane supports is expected to be hydrophilic. The water contact angle (Figure 2c, Table S1) of supports ranged from $106^{\circ}$ to $113^{\circ}$. The increased contact angle can be attributed to the rough surface of the support. Note that the water contact angle of bamboo fibers can vary greatly from $14^{\circ}$ to $72^{\circ}$ depending on the source and form of bamboo material. ${ }^{35,36}$ Therefore, the hydrophilicity of the support can be fine-tuned by employing bamboo fibers from different source.

The thermal stability of membrane supports was assessed by TGA (Figure 4). The TGA plots of bamboo fibers and PLA are in accordance with literature data. ${ }^{29}$ The TGA of bamboo fibers are divided into two steps. The first decomposition step occurs from $250{ }^{\circ} \mathrm{C}$ to $365{ }^{\circ} \mathrm{C}$, which is attributed to the thermal degradation of hemicellulose and the partial degradation of lignin. The second step from $365{ }^{\circ} \mathrm{C}$ to $520^{\circ} \mathrm{C}$ originates from the incineration of remaining lignin, char or fixed carbon. The decomposition of PLA occurs in one step at $300-380{ }^{\circ} \mathrm{C} .{ }^{30}$ The prepared membrane supports exhibit similar thermal stability to the bamboo fibers and PLA. The decomposition of membrane supports occurs in two steps, in which the first $\left(250-380^{\circ} \mathrm{C}\right)$ attributes to the decomposition of PLA and partial decomposition of lignin, while the second step originates from the combustion of remaining bamboo char. The TGA thermogram of PBI is in accordance with the spectra reported in the literature..$^{19}$

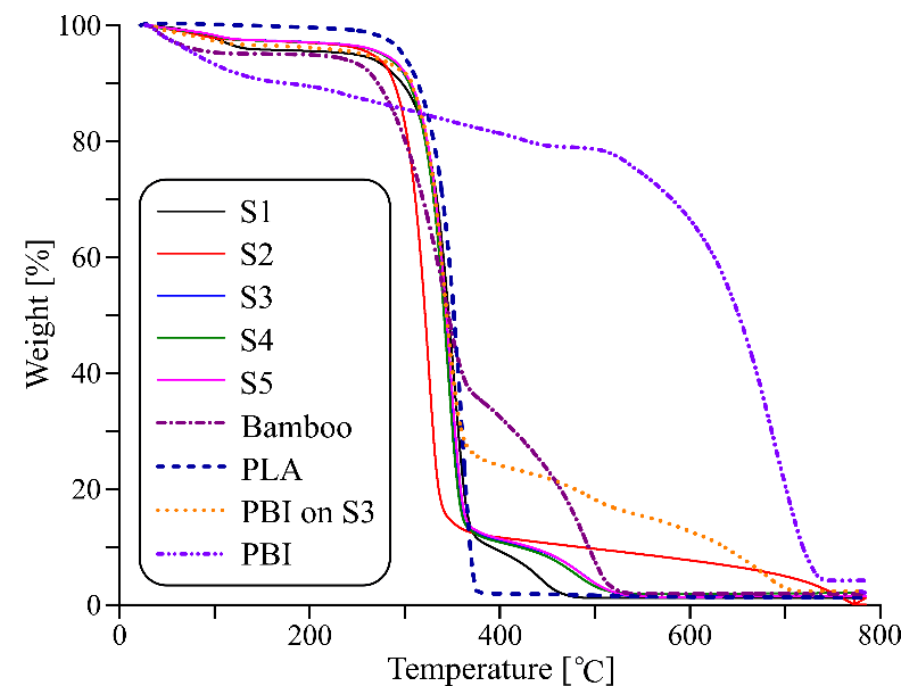

Figure 4. TGA thermograms of various materials used in this study.

The tensile strength and elongation are important parameters that describe the mechanical strength of the membrane supports (Figure 5). A linear correlation between the mechanical properties of the supports and the bamboo fiber/PLA ratio was found. The tensile strength increased by $124 \%$ from $32.7 \mathrm{MPa}$ to 73.3 $\mathrm{MPa}$ with the increasing bamboo content, while the elongation at break remained virtually the same in the range of $1.71 \%$ to $1.79 \%$. The tensile strength of bamboo/PLA membrane supports is comparable to the nonwoven membrane supports made from PET (29.4 MPa) and PE/PP (36-56 MPa). ${ }^{37,38}$ 


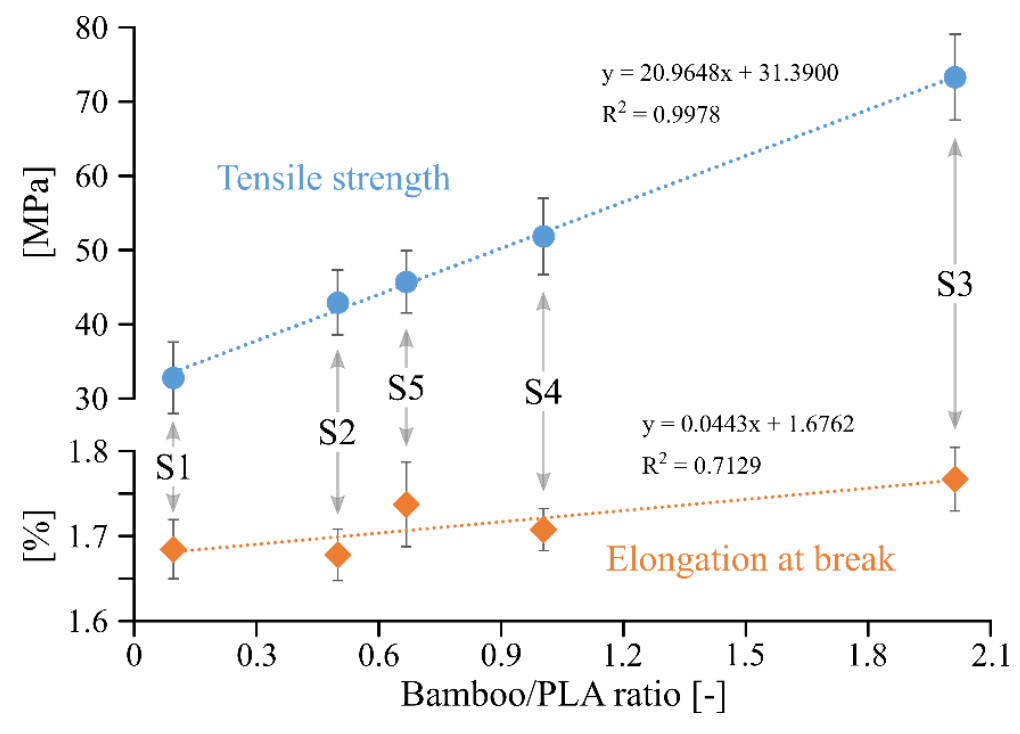

Figure 5. Tensile strength and elongation at break as a function of bamboo fiber/PLA mass ratio for the membrane supports.

Chemical stability of the membrane supports was tested in various classes of solvents: polar aprotic solvents, alcohols, esters, ethers, ketone, alkanes, aromatics, halogenated solvents, and water (Table 2). They were found to be stable in most solvents over 6 months. However, the materials disintegrated in halogenated solvents (chloroform and dichloromethane), and in some of the polar aprotic solvents such as DMC, THF, DMF and acetonitrile. Surprisingly, the membrane supports were found to be stable some conventional (DMAc, DMSO, NMP) and emerging green (PC, Cyrene ${ }^{\mathrm{TM}}, \gamma$-valerolactone and MeTHF) polar aprotic solvents. The solvent resistance of membrane support is in line with the solubility of PLA in the respective solvents. The stability and solvent resistance of the membrane supports could be further increased by crosslinking using biodegradable maleic anhydride. ${ }^{39}$

Table 2. Stability of the bamboo/PLA membrane supports in different classes of organic solvents: intact (I) and disintegrated (D). Values in parenthesis are the Hansen solubility parameters (HSP). ${ }^{40,41,42}$

\begin{tabular}{lccc}
\multicolumn{1}{c}{ Solvent } & $\begin{array}{c}\text { HSP } \\
\left(\mathbf{M P a}^{0.5}\right)\end{array}$ & Day $\mathbf{1}$ & Day $\mathbf{1 8 0}$ \\
\hline Polar aprotic: & & & \\
\hline PC & 27.2 & $\mathrm{I}$ & $\mathrm{I}$ \\
DMC & 20.2 & $\mathrm{D}$ & \\
Cyrene $^{\mathrm{TM}}$ & 22.7 & $\mathrm{I}$ & $\mathrm{I}$ \\
Y-Valerolactone & 23.7 & $\mathrm{I}$ & $\mathrm{I}$ \\
DMSO & 26.7 & $\mathrm{I}$ & $\mathrm{I}$ \\
2-MeTHF & 18.1 & $\mathrm{I}$ & $\mathrm{I}$ \\
THF & 19.5 & $\mathrm{D}$ & \\
DMF & 24.9 & $\mathrm{D}$ & \\
DMAc & 22.8 & $\mathrm{I}$ & $\mathrm{I}$ \\
NMP & 23.0 & $\mathrm{I}$ & $\mathrm{I}$ \\
Acetonitrile & 24.4 & $\mathrm{D}$ & \\
\hline Alcohols: & & & \\
\hline 1-butanol & 23.2 & $\mathrm{I}$ & $\mathrm{I}$
\end{tabular}




\begin{tabular}{llll} 
Ethanol & 26.5 & $\mathrm{I}$ & $\mathrm{I}$ \\
Isopropanol & 23.6 & $\mathrm{I}$ & $\mathrm{I}$ \\
Methanol & 29.6 & $\mathrm{I}$ & $\mathrm{I}$ \\
\hline Esters: & & & \\
\hline Ethyl lactate & 21.7 & $\mathrm{I}$ & $\mathrm{I}$ \\
Ethyl acetate & 18.2 & $\mathrm{I}$ & $\mathrm{I}$ \\
\hline Ethers: & & & \\
\hline CPME & 17.8 & $\mathrm{I}$ & $\mathrm{I}$ \\
MTBE & 16.2 & $\mathrm{I}$ & $\mathrm{I}$ \\
\hline Ketones: & & & \\
\hline Acetone & 19.9 & $\mathrm{I}$ & $\mathrm{I}$ \\
\hline Alkanes: & & & \\
\hline Heptane & 15.3 & $\mathrm{I}$ & $\mathrm{I}$ \\
Hexane & 14.9 & $\mathrm{I}$ & $\mathrm{I}$ \\
\hline Aromatics: & & & \\
\hline Toluene & 18.2 & $\mathrm{I}$ & $\mathrm{I}$ \\
\hline Halogenated: & & & \\
\hline Chloroform & 18.9 & $\mathrm{D}$ & \\
DCM & 19.8 & $\mathrm{D}$ & \\
\hline Water & 42.3 & $\mathrm{I}$ & $\mathrm{I}$ \\
\hline
\end{tabular}

Further to the chemical stability test, the membrane supports were also characterized by their swelling in some commonly used solvents including DMAc, NMP, PC, methanol, ethyl acetate, heptane, toluene and water. The swelling of membrane supports varies according to the polarity of the solvent and the bamboo content (Figure 6). The swelling of S1-5 as a function of solvent polarity followed a saturation curve: higher solvent polarity, resulted in greater swelling of membrane support. The swelling in heptane (least polar solvent) was found to be as low as $0.5 \%$ for all the supports, while the swelling in water (most polar solvent) varied between $5 \%$ and $9.9 \%$. The increase in bamboo content resulted in decreasing swelling ratio in all the solvents. For instance, the swelling in water decreased from $9.9 \%$ to $5 \%$ as the bamboo fiber/PLA mass ratio increased from 0.1 (S1) to 2 (S3). Therefore, the swelling of membrane supports can be controlled by varying the mass ratio of bamboo/PLA. 


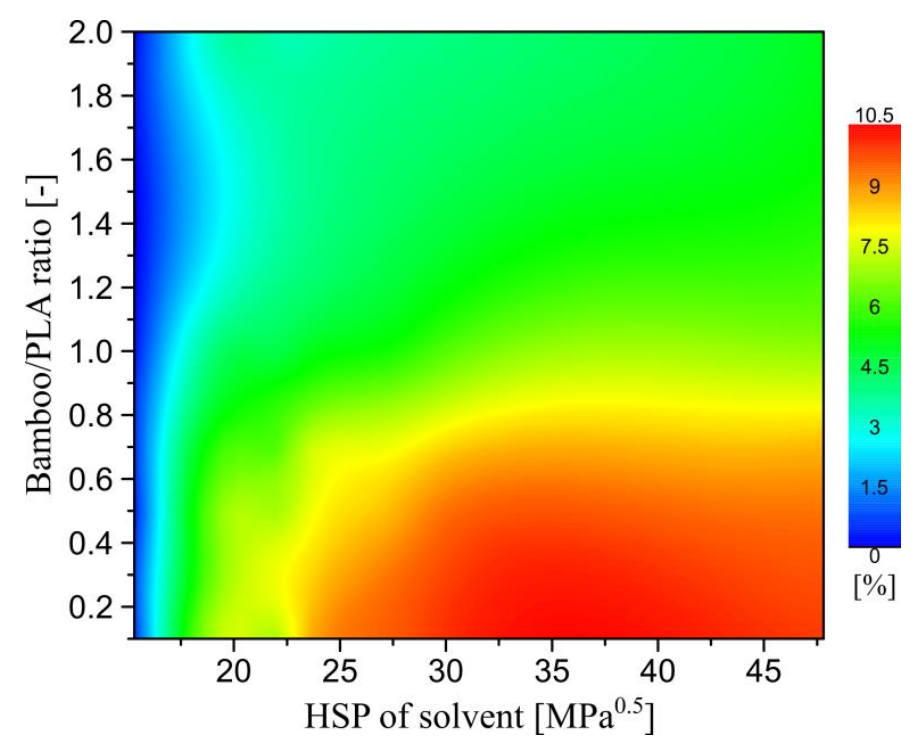

Figure 6. Linear swelling ratio for the different membrane supports as a function of Hansen Solubility Parameter and bamboo/PLA mass ratio. Refer to the Supporting Information for the data for each support and solvent.

The solvent permeance of S1-5 was studied in water, methanol, acetone and heptane covering a wide range of polarity (Figure 7a). Increasing solvent polarity and increasing bamboo content resulted in increasing permeance, which culminated at $1068 \pm 32 \mathrm{~L} \mathrm{~m}^{-2} \mathrm{~h}^{-1}$ bar ${ }^{-1}$ for S3 in water. The long-term stability and filtration performance of S3, both as a stand-alone support and with a PBI membrane cast on top of it, were successfully demonstrated over two weeks (Figure 7b). The supports were tested at 1 bar due to their high flux, while the PBI membrane on S3 required 10 bar as it falls within the nanofiltration range. The filtration with a membrane on top, and at higher pressure, both successfully demonstrated the viability of applying the non-woven material as a membrane support. 

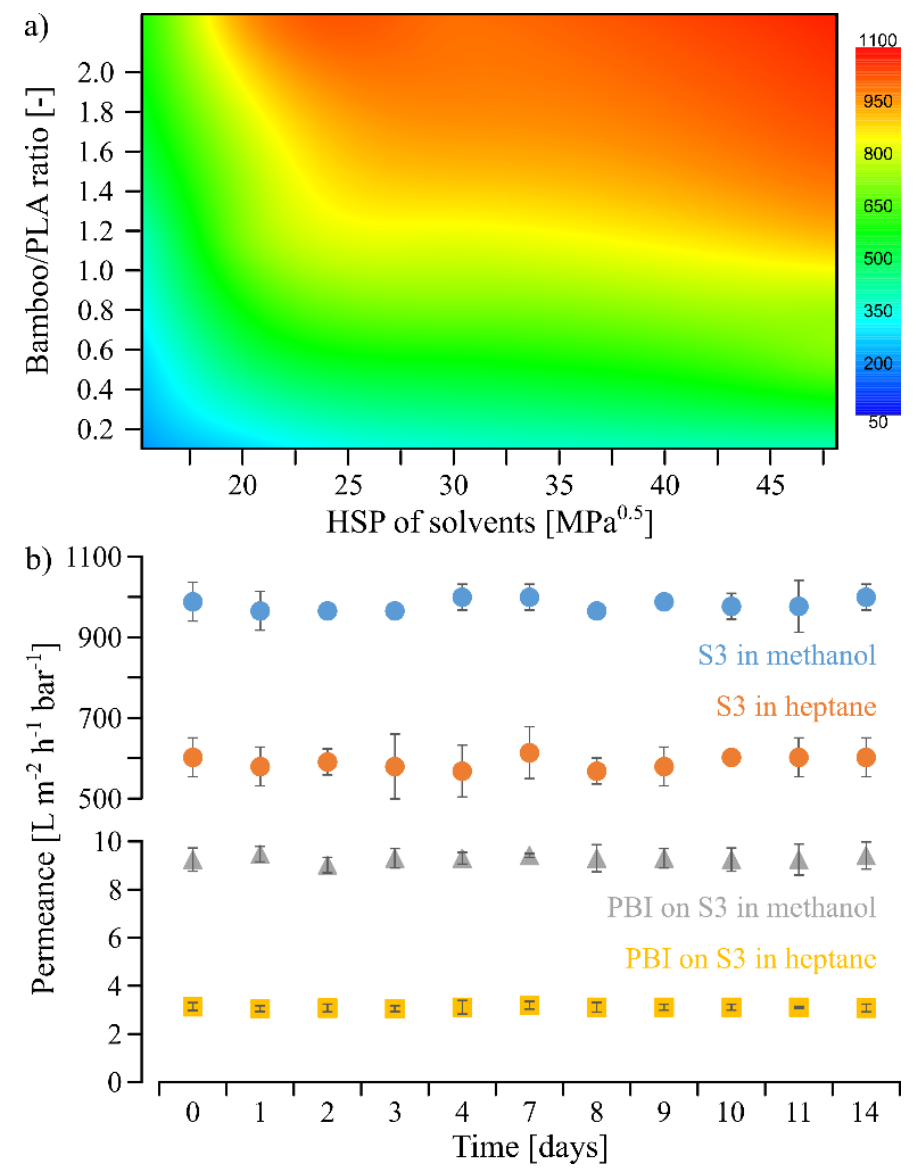

Figure 7. Mapping the permeance in water and various organic solvents at 1 bar as a function of Hansen solubility parameter (HSP) and bamboo fiber/PLA content (a), and long-term stability of S3 (1 bar) and PBI on S3 (10 bar) in methanol and heptane. All experiments were carried out in cross-flow configuration. The colormap represents the permeance expressed as $\mathrm{L} \mathrm{m}^{-2} \mathrm{~h}^{-1}$ bar $^{-1}$. Refer to the Supporting Information for the data for each support and solvent.

\section{Conclusions}

Membranes will only be truly sustainable when the environmental burden of their fabrication is mitigated by minimizing the use of toxic solvents and non-degradable, petroleum-based raw materials. In this work, a membrane support was fabricated from three renewable, non-toxic, biodegradable materials: bamboo fiber, PLA and DMC. The prepared composite film, in which the PLA served as a glue to adhere the bamboo fibers together, resulted in porous structure with rough surface that are attractive features for membrane supports. The tensile strength of the bamboo/PLA composites ranged from $33 \mathrm{MPa}$ to $73 \mathrm{MPa}$, which is comparable to the tensile strength of conventional PP and PET membrane supports. The mechanical strength of membrane supports was enhanced by increasing the bamboo fiber content. Increased bamboo/PLA ratio also resulted in decreased swelling by up to $50 \%$ and increased permeance, culminating at $1068 \pm 32 \mathrm{~L} \mathrm{~m}^{-2} \mathrm{~h}^{-1} \mathrm{bar}^{-1}$ in water. The porosity and the volume occupied by needle shaped particles were found to be 0.719 and $95 \%$, respectively.

The chemical stability of membrane supports were tested in 25 organic solvents and in water. Despite being unstable in some of the polar aprotic solvents (DMC, THF, DMF, acetonitrile), the membrane supports remained intact in DMAc, NMP and in emerging polar aprotic green solvents, such as Cyrene, PC, $\mathrm{v}$ - 
valerolactone and 2-MeTHF. Bamboo fibers were insoluble in all solvents providing great chemical stability, while the disintegration of membrane supports in some solvents originated from the dissolution of PLA. The membrane supports exhibited long-term stability, demonstrated over 6 months by soaking, and 2 weeks in continuous cross-flow filtration experiments.

The bamboo/PLA membrane supports could provide a green alternative for conventional membrane backing materials because they eliminate the need for petroleum-based polymers, such as PP, and toxic solvents, such as DMAc and NMP. The presented methodology can open new horizons for fabricating porous materials that are not limited to membrane supports but could include microfiltration membranes and adsorbents.

\section{Associated content}

\section{Supporting Information}

The Supporting Information is available free of charge on the ACS Publication website at DOI:

Detailed FTIR analyses; 3D reconstruction image and video, pore size distribution, white light interferometry images, water contact angle and RMS roughness data of membrane supports; SEM and AFM images of PBI membrane; nanofiltration process scheme; swelling ratio and permeance data for each solvent and support.

\section{Author information}

\section{Corresponding author}

*E-mail: gyorgy.szekely@manchester.ac.uk; gyorgy.szekely@kaust.edu.sa

\section{ORCID}

Hai Anh Le Phuong: 0000-0003-1380-4799

Nor Amira Izzati Ayob: 0000-0002-3369-7006

Christopher F. Blanford: 0000-0002-0112-7818

Nurul Fazita Mohammad Rawi: 0000-0001-9979-2295

Gyorgy Szekely: 0000-0001-9658-2452

\section{Notes}

The authors declare no competing financial interest.

\section{Acknowledgements}

The authors thank the experimental support provided by Dr Ben Spencer (white light interferometer) and Mr Abdulaziz Alammar (TGA) both from the University of Manchester, and Dr Wei Xu and Dr Sang-Hee Park (confocal microscopy) from the King Abdullah University of Science and Technology (KAUST). Fruitful discussions with Mr Levente Cseri from the University of Manchester is greatly acknowledged. The authors gratefully acknowledge Dr Azniwati Abdul Aziz for her advice on the preparation method of bamboo/PLA composites. HALP acknowledges the UK's Engineering and Physical Sciences Research Council (EPSRC) for funding her doctoral studies through the University of Manchester's doctoral training account administered by their School of Materials. Figure 1 and the graphical abstract were created by Heno Hwang, scientific illustrator at KAUST. 


\section{Abbreviations}

AFM, atomic force microscopy; CPME, cyclopentyl methyl ether; DCM, dichloromethane; DMAc, N,Ndimethyl acetamide; DMC, dimethylcarbonate; DMF, $\mathrm{N}, \mathrm{N}$-dimethylformamide; DMSO, dimethyl sulfoxide; EtOAc, ethyl acetate; HSP, Hansen solubility parameter; IPA, isopropanol; LSCM, laser scanning confocal microscopy ; MeCN, acetonitrile; $\mathrm{MeOH}$, methanol; 2-MeTHF, 2-methyl tetrahydrofuran; MTBE, methyl $t$ butyl ether; NMP, 1-methyl-2-pyrrolidone; PBI, polybenzimidazole; PC, propylene carbonate; PE, polyethylene; PET, polyethylene terephthalate ; PLA, poly(lactic acid); PP; polypropylene; RMS, root mean square; SEM, scanning electron microscopy; THF, tetrahydrofuran; WCA, water contact angle.

\section{References}

(1) Macedonio, F.; Drioli, E. Membrane Engineering for Green Process Engineering. Engineering 2017, 3, $290-298$.

(2) Szekely, G.; Jimenez-Solomon, M. F. ; Marchetti, P.; Kim, J. F. ; Livingston, A. G. Sustainability assessment of organic solvent nanofiltration: from fabrication to application. Green Chem. 2014, 16, 4440-4473.

(3) Razali, M.; Kim, J. F.; Attfield, M.; Budd, P. M.; Drioli, E.; Lee, Y. M.; Szekely, G. Sustainable wastewater treatment and recycling membrane manufacturing. Green Chem. 2015, 17, 5196-5205.

(4) Wang, H. H.; Jung, J. T.; Kim, J. F.; Kim, S.; Drioli, E.; Lee, Y. M. A novel green solvent alternative for polymeric membrane preparation via nonsolvent-induced phase separation (NIPS). J. Membr. Sci. 2019, 574, 44-54.

(5) Kim, D. L.; Moreno, N.; Nunes, S. P. Fabrication of polyacrylonitrile hollow fiber membranes from ionic liquid solutions. Polym. Chem. 2016, 7, 113-124.

(6) Rasool, M. A.; Vankelecom, I. F. J. Use of $y$-valerolactone and glycerol derivatives as bio-based renewable solvents for membrane preparation. Green Chem. 2019, 21, 1054-1064.

(7) Tham, H. M.; Japip, S.; Hua, D.; Chung, T.-S. Green Layer-by-Layer Method for the Preparation of PolyacrylonitrileSupported Zinc Benzene-1,4-dicarboxylic Acid Membranes. ChemSusChem 2018, 11, 2612-2619.

(8)Galiano, F.; Briceno, K.; Marino, T.; Molino, A.; Christensen, A. K.; Figoli, A. Advances in biopolymer-based membrane preparation and applications. J. Membr. Sci. 2018, 564, 562-586.

(9) Farah, S.; Anderson, D. G.; Langer, R. Physical and mechanical properties of PLA, and their functions in widespread applications - A comprehensive review. Adv. Drug Delivery Rev. 2016, 107, 367-392.

(10) John, G.; Nagarajan, S.; Vemula, P. K.; Silverman, J. R.; Pillai, C.K.S. Natural monomers: A mine for functional and sustainable materials - Occurrence, chemical modification and polymerization. Prog. Polym. Sci. 2019, 92, $158-209$.

(11) Galiano, F.; Briceno, K.; Marino, T.; Molino, A.; Christensen, A. K.; Figoli, A. Advances in biopolymer-based membrane preparation and applications. J. Membr. Sci. 2018, 564, 562-586.

(12) Shalwan, A.; Yousif, B. F. In State of Art: Mechanical and tribological behaviour of polymeric composites based on natural fibres. Mater. Des. 2013, 48, 14-24.

(13) Delgado-Aguilar, M.; Julian, F.; Tarres, Q.; Mendez, J. A.; Mutje, P.; Espinach, F. X. Bio composite from bleached pine fibers reinforced polylactic acid as a replacement of glass fiber reinforced polypropylene, macro and micromechanics of the Young's modulus. Composites Part B 2017, 125, 203-210.

(14) Gu, F.; Zheng, Y.; Zhang, W.; Yao, X.; Pan, D.; Wong, A. S. M.; Guo, J.; Hall, P.; Sharmin, N. Can bamboo fibres be an alternative to flax fibres as materials for plastic reinforcement? A comparative life cycle study on polypropylene/flax/bamboo laminates. Ind. Crop Prod. 2018, 121, 372-387.

(15) Porras, A.; Maranon, A. Development and characterization of a laminate composite material from polylactic acid (PLA) and woven bamboo fabric. Composites Part B 2012, 43, 2782-2788.

(16) Qian, S.; Zhang, H.; Yao, W.; Sheng, S. Effects of bamboo cellulose nanowhisker content on the morphology, crystallization, mechanical, and thermal properties of PLA matrix biocomposites. Composites Part B 2018, 133, 203209.

(17) Zuo, Y.; Li, W.; Li, P.; Liu, W.; Li, X.; Wu, Y. Preparation and characterization of polylactic acid-g-bamboo fiber based on in-situ solid phase polymerization. Ind. Crop Prod. 2018, 123, 646-653.

(18) Yusoff, R. B.; Takagi, H.; Nakagaito, A. N. Tensile and flexural properties of polylactic acid-based hybrid green composites reinforced by kenaf, bamboo and coir fibers. Ind. Crop Prod. 2016, 94, 562-573.

(19) Fei, F.; Le Phuong, H. L.; Blanford, C. F.; Szekely, G. Tailoring the Performance of Organic Solvent Nanofiltration Membranes with Biophenol Coatings. ACS Appl. Polym. Mater. 2019, 1, 452-460. 
(20) Ignacz, G.; Fei, F.; Szekely, G. Ion-Stabilized Membranes for Demanding Environments Fabricated from Polybenzimidazole and Its Blends with Polymers of Intrinsic Microporosity. ACS Appl. Nano Mater. 2018, 1, 6349-6356. (21) Huang, L.-K.; Wang, M.-J. J. Image thresholding by minimizing the measure of fuzziness. Pattern Recognit. 1995, 28 41-51.

(22) Ridler, T. W.; Calvard, S. Picture thresholding using an iterative selection method. IEEE Trans. Syst. Man Cybern. 1978, 8, 630-632.

(23) Li, C. H.; Tam, P. K. S. An Iterative Algorithm for Minimum Cross Entropy Thresholding. Pattern Recognit. Lett. 1998, $18,771-776$.

(24) Schmid, B.; Schindelin, J.; Cardona, A.; Longair, M.; Heisenberg, M. A high-level 3D visualization API for Java and ImageJ. BMC Bioinf. 2010, 11, 274-280.

(25) Doube, M.; Kłosowski, M. M.; Arganda-Carreras, I.; Cordeliéres, F.; Dougherty, R. P.; Jackson, J.; Schmid, B.; Hutchinson, J. R.; Shefelbine, S. J.; BoneJ: free and extensible bone image analysis in ImageJ. Bone 2010, 47, 1076-1079. (26) Dougherty, R.; Kunzelmann, K.-H. Computing Local Thickness of 3D Structures with ImageJ. Microsc. Microanal. 2007, 13, 1678-1679.

(27) Hildebrand, T.; Rüegsegger, P. A new method for the model-independent assessment of thickness in threedimensional images. J. Microsc. 1997, 185, 67-75.

(28) Fei, F.; Cseri, L.; Szekely, G.; Blanford, C. F. Robust Covalently Cross-linked Polybenzimidazole/Graphene Oxide Membranes for High-Flux Organic Solvent Nanofiltration. ACS Appl. Mater. Interfaces 2018, 20, 16140-16147. (29)Johari, A. P.; Mohanty, S.; Kurmvanshi, S. K.; Nayak, S. K. Influence of Different Treated Cellulose Fibers on the Mechanical and Thermal Properties of Poly(lactic acid). ACS Sustainable Chem. Eng. 2016, 4, 1619-1629.

(30) Li, M.-F.; Shen, Y.; Sun, J.-K.; Bian, J.; Chen, C.-Z.; Sun, R.-C. Wet Torrefaction of Bamboo in Hydrochloric Acid Solution by Microwave Heating. ACS Sustainable Chem. Eng. 2015, 3, 2022-2029.

(31) Galiano, F.; Ghanim, A. H.; Rashid, K. T.; Marino, T.; Simone, S.; Alsalhy, Q. F.; Figoli, A. Preparation and characterization of green polylactic acid (PLA) membranes for organic/organic separation by pervaporation. Clean Technol. Environ. Policy 2019, 21, 109-120.

(32) Holda, A.K.; Vankelecom, I. F. J. Understanding and guiding the phase inversion process for synthesis of solvent resistant nanofiltration membranes. J. Appl. Polym. Sci. 2015, 132, 42130-42146.

(33) Onder, O. C.; Nazeer, M. A.; Yilgor, E.; Yilgor, I. Spontaneous formation of microporous poly(lactic acid) coatings. Prog. Org. Coat. 2018 125, 249-256.

(34) Anwat, U. Paridah, M. T.; Hamdan, H.; Zaidon, A.; Roziela Hanim, A.; Nordahlia, A. S. Adhesion and Bonding Properties of Low Molecular Weight Phenol Formaldehyde-Treated Plybamboo. J. Trop. For. Sci. 2012, 24, 379-386.

(35) Wang, F.; Li, S.; Wang, L. Fabrication of artificial super-hydrophobic lotus leaf-like bamboo surfaces through soft lithography. Colloids Surf., A 2017, 513, 389-395.

(36) Chen, H.; Zhang, W.; Wang, X.; Wang, H.; Wu, Y.; Zhong, T.; Fei, B. Effect of alkali treatment on wettability and thermal stability of individual bamboo fibers. J. Wood Sci. 2018, 64, 398-405.

(37) He, H.; Wang, X.; Liu, W. Effects of PEGDMA on a PET non-woven fabric embedded PAN lithium-ion power battery separator. Solid State Ionics 2016, 294, 31-36.

(38) Jang, K.-S. Exploring polyethylene/polypropylene non-woven fabrics derived from twodimensionally co-extruded composites: Effects of delamination, consolidation, drawing and nanoparticle incorporation on mechanics, pore size and permeability. Compos. Sci. Technol. 2018, 165, 380-387.

(39) Gonzalez-Lopez, M. E.; Robledo, Ortiz, J. R.; Manriquez-Gonzalez R.; Silva-Guzman, J. A., Perez-Fonseca, A. A. Polylactic acid functionalization with maleic anhydride and its use as coupling agent in natural fiber biocomposites: $A$ review. Compos. Interfaces 2018, 25, 515-538.

(40) Hansen, C. M. Hansen Solubility Parameters, A User's Handbook CRC press:Boca Raton, 2007; pp. 345-483.

(41) Sherwood, J.; De bruyn, M.; Constantinou, A.; Moity, L.; McElroy, C. R.; Farmer, T. j.; Duncan, T.; Raverty, W.; Hunt, A. J.; Clark, J. H. Dihydrolevoglucosenone (Cyrene) as a bio-based alternative for dipolar aprotic solvents. Chem. Commun. 2014, 50, 9650-9652.

(42) Sanchez-Camargo, A. P.; Montero, L.; Cifuentes, A.; Herrero, M.; Ibanez, E. Application of Hansen solubility approach for the subcritical and supercritical selective extraction of phlorotannins from Cystoseira abies-marina. RSC Adv. 2016, 6, 94884-94895. 